\title{
Evaluación alternativa: Separar el grano de la paja
}

\author{
Sheila W. Valencia
}

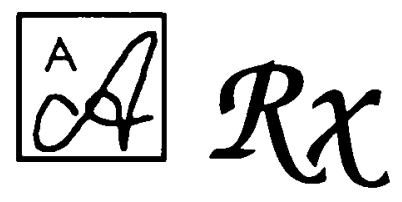

Por su significatividad respecto a la tendencia predominante sobre lo que se está buscando como alternativa para la evaluación, incluimos un editorial de The Reading Teacher comentando los objetivos y requisitos de esa evaluación que concentraría lo mejor de las dos grandes tendencias formal e informal.

Durante los últimos cinco años hemos asistido a una oleada de interés en evaluaciones alternativas de la lectura. Muchos han escrito sobre los problemas de la evaluaciones existentes y muchos han intentado superar estos obstáculos proponiendo alternativas. La motivación para estas alternativas es doble: En primer lugar, nos hemos hartado de hacer malabarismos con la enseñanza y la evaluación tratando de conjugar lo que, con frecuencia, son dos objetivos contrapuestos. Necesitamos un sistema de evaluación que satisfaga la alineación de enseñanza y evaluación para que tanto nosotros como nuestros estudiantes podamos ser verdaderamente responsables de los resultados educativos importantes.

En segundo lugar, deseamos comunicar a estudiantes, padres, administradores y a nosotros mismos, los de dentro y fuera del aula, el logro real de nuestros estudiantes en lectoescritura. Deseamos trazar una imagen más compleja de la lectoescritura y relatar una historia más completa de lo que hasta ahora hemos podido hacer con las medidas existentes. Con metas tan loables, no es de sorprender que la evaluación alternativa haya despertado tanto interés.

Existen pocas dudas de que ninguno de nosotros se escapará de estos nuevos enfoques: Llegarán en forma de nuevas pruebas estanda- 
rizadas, listas de chequeo y observaciones de enseñantes, y evaluaciones de programas de instrucción por parte de editores, para nombrar solo unos cuantos. Pero quizá el cambio más drástico que hemos empezado a ver y que seguiremos viendo es un énfasis renovado en la evaluación realizada en clase.

A los escépticos en relación a esta nueva tendencia les preocupa la evaluación en clase. Nos recuerdan que la razón de que las pruebas estandarizadas se hicieran tan populares fue que las notas, los diplomas y las evaluaciones hechas en clase no se consideraban fiables. La gente tenía poca fe en estas evaluaciones porque los resultados variaban de un enseñante a otro y de una escuela a otra: No eran dignas de confianza. No había una manera clara de comunicar los logros de nuestros alumnos a otras personas. Esto condujo a una generación extraordinaria de evaluaciones de clase poco fiables que parecían minipruebas estandarizadas de rendimiento, con lo que los problemas de las pruebas estandarizadas arraigaron aún más en el sistema.

A diferencia de las evaluaciones en clase del pasado que reflejaban pruebas tradicionales y modelos de evaluación publicados, esta nueva tendencia nos encuentra observando con más atención la fiabilidad de las tareas de evaluación y su alineación con la investigación, la teoría y las prácticas educativas actuales. Además, nos recuerda que el aprendizaje es demasiado complejo y la evaluación demasiado imperfecta como para basarnos en un solo índice de éxito.

Las estrategias de evaluación que están ganando impulso vienen en muchas formas y bajo muchas etiquetas como evaluación basada en el rendimiento, carpetas, listas de chequeo, evaluación holística y "observación infantil". La base conceptual que subyace a todas estas estrategias es que deberíamos considerar a los estudiantes responsables de los logros y las disposiciones que hemos diseñado como esenciales e importantes en la aptitud para la lectura, y someter a prueba estos resultados en contextos que preserven la integridad de las tareas de lectoescritura. Sin embargo, cambiar a tareas de lectura más válidas no garantiza en sí mismo una evaluación de calidad. Y aquí es donde debemos separar el grano de la paja.

\section{LA BUENA EVALUACION}

El concepto que hay detrás de una "buena" evaluación de la lectura, tanto si se trata de una prueba estandarizada como si es una evaluación alternativa de clase,es el mismo. En primer lugar, debe ser válida; debe evaluar lo que hemos definido como lectura verdarera y valoramos como tal. En segundo lugar, debe ser fiable; debe tener procedimientos claramente establecidos para recopilar información (por ejemplo, observaciones, trabajos escritos, entrevistas, etc.) y para evaluar la calidad de esa información. A medida que vayamos adoptando más evaluaciones de clase, deberemos ser capaces de articular y demostrar que nuestras evaluaciones cumplen con estos criterios de una "buena" evaluación. 
Validez. La necesidad de validez es de lo más evidente; es el punto más fuerte del movimiento por la evaluación alternativa. Ahora, más que en cualquier época del pasado, la evaluación de la lectoescritura empieza a parecerse a la auténtica lectura y escritura. Por ejemplo, es común ver evaluaciones alternativas de lectoescritura emergente en las que los enseñantes observan a los niños tomando libros, pasando páginas y "leyendo" a un amigo o a un animal de peluche favorito. También vemos evaluaciones en las que los estudiantes leen distinto tipos de textos (por ejemplo, narrativos, informativos, documentos) con fines distintos y en los que la variabilidad es esperada y fomentada, en vez de desaconsejada. En otras evaluaciones, los estudiantes escriben o discuten lo que han leído y los procesos y productos de las actividades se utilizan para evaluar el rendimiento.

Muchas evaluaciones alternativas propuestas en la actualidad poseen este atributo de validez. No es algo trivial. En realidad, representa un primer paso esencial en tanto que evaluamos los objetivos importantes de la enseñanza de la lectoescritura y tomamos decisiones sobre el qué y el cómo en la evaluación del rendimiento.

Fiabilidad. Las evaluaciones formales suelen dar fe de su "buena" calidad apelando a datos estadísticos que apoyan su fiabilidad. Sin embargo, la mayoría de las evaluaciones de clase alternativas aún no se han dirigido a este aspecto. Aunque estos tipos de evaluación no están estandarizados en el sentido estadístico de la palabra, deben tener alguna normativa. Carecer de normas o criterios equivale a salir a navegar sin cartas de navegación. No habrá manera de determinar dónde estamos, hasta dónde hemos llegado y cómo debemos seguir. Observar a un niño mientras lee o examinar un escrito sin tener una sensación clara de cómo se deben interpretar las conductas o evaluar el rendimiento es probable que provoque incomodidad en enseñante y alumno, e incluso es más probable que lleve a una falta de fiabilidad.

-- El peligro de nuevas evaluaciones no fiables es que las evaluaciones alternativas pueden no llegar a obtener la credibilidad que merecen; es posible que las únicas personas que tengan confianza en estas medidas sean los individuos que utilizan cada estrategia de evaluación. No seremos capaces de comunicarnos con otros fuera de nuestras aulas o escuelas. Además, sin expectativas y normativas claramente articuladas, incluso estas personas que utilizan las evaluaciones pueden encontrarse preguntándose en qué deben centrarse cada vez que llevan a cabo una evaluación. La cuestión no estriba en imponer más formalidad a la evaluación alternativa sino más bien en ser claro sobre las expectativas y las normas. Podemos mantener normativas sin imponer una estandarización.

Las evaluaciones fiables requerirán unas normativas claras. Este será el próximo paso crucial que deberemos dar. La claridad procederá de nosotros, los enseñantes de lectoescritura, que conocemos la investigación y la instrucción, poseemos una valiosa experiencia práctica con niños, desarrollamos competencia en crear, implementar y evaluar evaluaciones alternativas y participamos en un diálogo continuo con nues- 
tros colegas para establecer una visión compartida. Confrontar los temas de las expectativas y las normativas es esencial para que las evaluaciones alternativas sean útiles y significativas.

Será con este espíritu de la "buena" evaluación alternativa como se escribirá esta columna durante el próximo año. He reunido un grupo de educadores de lectura que han diseñado y sometido a prueba evaluaciones alternativas. Describirán los fundamentos y los procedimientos que han usado para evaluar el progreso de los alumnos, a veces en régimen individual, a veces en grupo. El objetivo de esta columna es ofrecer a los enseñantes que trabajan en clase una firme comprensión de las características de la buena evaluación alternativa ofreciendo los esfuerzos realizados por otras personas. Al final, espero estimular el intercambio y el diálogo que son tan esenciales para que la evaluación alternativa llegue a ocupar el lugar que merece en el campo de la educación.

\section{Evaluación alternativa:}

separar el grano de la paja

Sheila W. Valencia

CL\&E, 1993, 19-20, 63-66

Datos sobre el autor: Sheila W. Valencia trabaja en la Universidad de Washington

Articulo original:Alternative assessment: Separating the wheat from the chaff. En The Reading Teacher, Vol. 44, No 1, september 1990, pp. 60-61. Traducción de Genis Sánchez. Reproducido con autorización deSheila W. Valencia y de la International Reading Asociation (La I.R.A. no se responsabiliza de la adecuación de la traducción)

Dirección: University of Washington, 122 Miller Hall DQ-12, Seattle, WA 98195, USA

(1) De todos los artículos deberá solicitarse por escrito autorización de CL\&E y de los autores para el uso en forma de facsímil, fotocopia o cualquier otro medio de reproducción impresa. CL\&E se reserva el derecho de interponer acciones legales necesarias en aquellos casos en que se contravenga la ley de derechos de autor. 